\title{
L'inflation à la mode Kayapo : rituel, marchandise et monnaie chez les Xikrin (Kayapo) de l'Amazonie brésilienne
}

\section{César Gordon}

\section{CpenEdition}

\section{Journals}

Édition électronique

URL : https://journals.openedition.org/jsa/11567

DOI : 10.4000/jsa. 11567

ISSN : 1957-7842

Éditeur

Société des américanistes

Édition imprimée

Date de publication : 5 décembre 2010

Pagination : 205-228

ISSN : 0037-9174

Référence électronique

César Gordon, «L'inflation à la mode Kayapo : rituel, marchandise et monnaie chez les Xikrin

(Kayapo) de l'Amazonie brésilienne », Journal de la Société des américanistes [En ligne], 96-2 | 2010, mis en ligne le 10 décembre 2014, consulté le 03 septembre 2022. URL : http://journals.openedition.org/ jsa/11567 ; DOI : https://doi.org/10.4000/jsa.11567 


\title{
L'INFLATION À LA MODE KAYAPO : RITUEL, MARCHANDISE ET MONNAIE CHEZ LES XIKRIN (KAYAPO) DE L'AMAZONIE BRÉSILIENNE
}

\author{
César GORDON *
}

Les Xikrin d'Amazonie brésilienne (groupe kayapo de l'État du Para, de langue mebêngôkre appartenant à la famille linguistique ge) portent un intérêt très marqué aux objets produits par les Blancs, notamment à l'argent et aux biens industriels. Également frappante chez eux est la notable implication de ces objets dans tous les domaines de la vie indigène : parenté, économie, politique et rituel. Nous nous attachons à démontrer que l'incorporation de marchandises dans la société xikrin ne doit pas être vue simplement comme un épiphénomène de l'histoire du contact, mais comme le résultat d'une interaction complexe entre les principes généraux de la socio-cosmologie indigène et les conditions historiques particulières dans lesquelles ces principes agissent et s'actualisent. [Mots-clés : Amazonie, Kayapo, rituel, marchandise, monnaie.]

Inflation Kayapo-style : ritual, commodity and money among the Xikrin (Kayapo) of Brasilian Amazonia. The Xikrin (a Kayapo group of Brazilian Amazonia, who speak the mebêngôkre language of the Gê linguistic stock) show a great interest in objects made by the White people. Surprisingly enough, money and goods have become embedded in every domain of Xikrin life, including kinship, economy, politics and ritual. In this paper, I try to show that this sort of consumerism results of a complex interaction between general principles of Kayapo sociocosmology and the particular historical conditions in which such principles operate and are actualized. [Key words : Amazonia, Kayapo, ritual, merchandise, money.]

Inflação à moda Kayapó : ritual, mercadoria e dinheiro entre os Xikrin (Kayapó) da Amazonia Brasileira. Os índios Xikrin da Amazônia (grupo Kayapó do Estado do Pará, falantes da língua mebêngôkre da família Jê) manifestam grande interesse pelos objetos produzidos pelos « brancos », especificamente ao dinheiro e às mercadorias. É notável a implicação desses objetos em todos os domínios da vida indígena: parentesco,

* Departamento de Historia e Economia, Universidade Federal Rural do Rio de Janeiro (UFRRJ), av. Governador Roberto Silveira, Moquetá, 26285-060, Nova Iguaçu, Brésil [cesar@cesargordon.net].

Journal de la Société des Américanistes, 2010, 96-2, pp. 205-228. O Société des Américanistes. 
economia, política e ritual. Este artigo demonstra que a incorporação de mercadorias na sociedade Xikrin não deve ser vista simplesmente como um epifenômeno da história do contato, mas como resultado da interação complexa entre os princípios gerais da sociocosmologia indígena e as condições históricas particulares nas quais tais princípios operam e se atualizam. [Palavras chave: Amazonia, Kayapó, ritual, mercadorias, dinheiro.]

\section{LE PAYSAGE RÉNOVÉ DE L'AMÉRICANISME}

La circulation de l'argent, les échanges mercantiles et la consommation de biens industrialisés sont des phénomènes de plus en plus présents et importants dans l'expérience contemporaine d'une grande partie des groupes indigènes brésiliens. La situation historique dans laquelle ces phénomènes apparaissent est liée au processus d'insertion de l'Amazonie dans les transformations contemporaines à grande échelle, nommées conventionnellement globalisation. Durant les trente dernières années, ce processus a agi en modifiant de manière accentuée le paysage géopolitique, culturel et humain de la région amazonienne et, en conséquence, les conditions d'existence des populations indiennes qui habitent cette région.

Au Brésil, le résultat visible de la globalisation est une plus grande intégration des Indiens à la vie politique, économique et culturelle du pays. Aujourd'hui, des interactions de plus en plus étendues et complexes se tissent entre les peuples indigènes et entre ces derniers et d'autres sujets ou institutions non indiennes, comme l'État et les marchés locaux ou internationaux, grâce au développement des possibilités de communication et de transport.

Les Indiens vivent une époque de changements rapides. Les ONG sont omniprésentes dans le quotidien indigène, de même que les associations et les projets économiques et culturels les plus variés tels que les écoles et universités, les églises évangéliques, le football, les élections, les permis de conduire, les comptes bancaires, la télévision, les ordinateurs, les caméras vidéos, le téléphone portable et internet... Bref, toute une série extrêmement variée de nouvelles connaissances et technologies, de nouveaux objets et moyens, de nouveaux discours et pratiques culturelles, envahit le monde indigène. Les Indiens, de leur côté, essayent de s'approprier ces nouveaux éléments à des fins politiques et économiques, mais surtout dans le cadre d'une finalité plus globale : la reproduction de leur mode de vie, tel qu'ils le conçoivent, le vivent et le transforment.

Pour utiliser la fameuse analogie thermodynamique de Lévi-Strauss (1973 [1960]), il semblerait que nous assistions à un processus de « réchauffement » des sociétés indiennes. Pour elles, de fait, les défis sont immenses. Il est nécessaire de rendre compte, de manière conceptuelle et pratique, de tout un univers de relations complexes et souvent contradictoires, caractéristique de la modernité ou de la post-modernité dans laquelle nous vivons. D'un autre côté, pour les 
ethnologues qui étudient les Indiens, cette reconfiguration du paysage amérindien fait apparaître de nouveaux défis, que ce soit par rapport à nos méthodes de recherche ou par rapport aux modèles théoriques que nous construisons et utilisons pour expliquer le monde indigène.

Dans les années 1980-1990, quelques auteurs, comme Bruce Albert (1988, 1993, 1997) en France, Stephen Hugh-Jones $(1988,1992)$ en Angleterre et Terence Turner (1993, 1995) aux États-Unis, avaient perçu la nécessité d'incorporer la question du changement ainsi que ce nouvel ensemble de phénomènes dans les investigations ethnologiques dites plus "classiques». Ils cherchaient des modèles d'explication alternatifs qui permettraient d'aller au-delà des études sur l'acculturation ou sur les contacts interethniques. Autrement dit, il fallait vérifier si les modèles, que les ethnologues américanistes avaient développés dans les années 1970 et 1980 pour analyser les mythes, la parenté, le chamanisme, la politique et les rituels indigènes, nous permettaient de comprendre la transformation actuelle de ces thèmes et son imbrication avec le monde non indien selon une échelle totalement nouvelle comme celle que nous voyons à présent (HughJones 1992, p. 43) ${ }^{1}$.

\section{Les Ge et les Kayapo dans CE Paysage}

Mon travail s'insère dans cette nouvelle thématique de recherche. Depuis 1998, j'étudie les Kayapo Xikrin de l'Amazonie brésilienne. Je me suis retrouvé confronté à l'un des aspects de ce nouveau paysage américaniste : précisément la question de la circulation de l'argent et la consommation des biens dans la société xikrin (Gordon 2006). Je n'avais pas particulièrement d'intérêt pour ce thème. J'étais allé sur le terrain pour étudier la parenté. Mais la question était si importante et intéressante pour les Indiens eux-mêmes, que cela aurait été un manque total de sensibilité ethnographique de ma part de ne pas me laisser guider par leurs intérêts.

Bien que la monétarisation et l'augmentation de la consommation soient désormais des phénomènes visibles chez un grand nombre de groupes indigènes, ces thèmes ne sont pas encore beaucoup abordés par les ethnologues ${ }^{2}$. Certains auteurs qui ont traité la question ne sont pas parvenus à échapper au paradigme de l'acculturation, selon lequel l'entrée de l'argent et des marchandises dans le monde indigène serait un élément profondément perturbateur et délétère parce qu'elle détruirait les liens tribaux traditionnels (Hoffmann 1964). Ou alors, de façon inverse et complémentaire, on tentait de justifier le phénomène comme la manifestation d'une affirmation autochtone d'autonomie économique et politique face à la société nationale (Turner 1992 ; Howard 2002). Entre acculturation et résistance, il restait peu d'espace pour une compréhension du phénomène d'un point de vue véritablement indigène, c'est-à-dire dans lequel le désir d'argent et de 
consommation n'apparaîtrait pas seulement comme une simple réaction - qu'elle soit positive ou négative - au contact, mais bien comme l'expression historique et conjoncturelle d'un certain régime de reproduction sociale.

Ainsi, mon travail est parti de la constatation du grand intérêt manifesté par les Xikrin pour les objets produits par les Blancs, notamment l'argent et les biens industriels, tout comme la notable implication de ces objets dans tous les domaines de la vie indigène : parenté, économie, politique et rituel. De manière inattendue, les objets mercantiles avaient acquis dans la société kayapo un statut de « fait social total ». En effet, pour aborder la parenté, il fallait parler aussi bien d'argent et de marchandises que de parents et d'alliés. Parallèlement, j'ai été amené à comprendre qu'il n'était pas possible de réfléchir sur l'utilisation de l'argent et sur la consommation des biens sans tenir compte de cette question : la relation des Xikrin avec le kuben (mot kayapo désignant l'étranger, le non-Indien, le Blanc). Cela m'a conduit à une réflexion plus générale sur le régime symbolique kayapo qui tenait compte des modes de relation avec l'altérité - c'est-à-dire avec des sujets définis par eux comme Autre, comme non-Kayapo - et, plus particulièrement, sur le thème de l'incorporation de l'Autre. J'ai essayé de comprendre comment la signification et la fonction des objets mercantiles dépendaient intrinsèquement des objets dits « traditionnels », les deux types d'objets pensés par les Kayapo comme ayant une origine exogène.

Le rôle constitutif de l'altérité dans les processus de prédation ou d'appropriation de capacités créatrices ou productives extérieures (au groupe local, à l'univers des parents et à la personne) a été démontré de manière consistante par de nombreux ethnologues américanistes au cours des deux ou trois dernières décennies (Albert 1985, 1988 ; Descola 1987, 1993 ; Erikson 1986 ; Fausto 2001 ; Menget 1985 ; Kaplan 1984 ; Vilaça 1992 ; Viveiros de Castro 1992, entre autres). Toutefois, les matériaux ethnographiques des populations de langue ge, comme les Kayapo, ne semblaient pas contribuer pour beaucoup à ces formulations. Ils étaient marqués par la problématique dualiste ou dialectique et par l'influence des analyses du groupe "Harvard-Museu Nacional Brasil Central» dirigé par Maybury-Lewis (1979). Plusieurs chercheurs, comme Turner, Melatti, da Matta, Carneiro da Cunha, Seeger, en ont fait partie. Le travail influent de Viveiros de Castro (1992) a contribué à renforcer le contraste entre les Ge et les Tupi (et Carib) et, d'une certaine manière, à isoler les premiers du restant du paysage amazonien.

Néanmoins, certains travaux sur les Kayapo - comme ceux de Verswijver (1992), Lea (1986) et Vidal Giannini (1991) et, même, de Seeger (1981) chez les Suya qui sont linguistiquement et culturellement proches des Kayapo - indiquaient qu'un rapprochement avec la problématique de la prédation amazonienne serait profitable. Dans le cas des Kayapo, la mythologie et les pratiques guerrières s'articulent autour de l'idée selon laquelle la constitution de la société (comme processus continu) dépendait, et dépend, de l'acquisition d'éléments 
obtenus de l'extérieur, c'est-à-dire originellement possédés par des non-humains ou des étrangers. Mais, et c'est mon argument, dans le cas des Kayapo l'expression empirique de la prédation comme forme prototypique de relation entre " moi » et « l'autre " ne s'exprime pas par le cannibalisme, par la chasse aux têtes ou par le chamanisme, mais au moyen de la capture d'objets et d'expressions objectivées, que nous pourrions qualifier d'artistiques, de techniques ou d'esthétiques, et du traitement symbolique de ces objets dans le système rituel ${ }^{3}$.

C'est justement cette analyse qui m'a permis de rapprocher les groupes kayapo du modèle de la prédation ontologique et des systèmes guerrierscannibales amazoniens. Elle m'offrait en effet la possibilité de reconfigurer la distinction entre les peuples ge du Brésil central et les peuples de la forêt dense, dont le contraste a été cristallisé par Viveiros de Castro (1992) dans son élaboration du modèle amazonien et, postérieurement, redéfini par Fausto (2001) en termes de régimes centripètes et centrifuges.

Le système kayapo fonctionne autant selon une logique appropriative (plus caractéristique des régimes guerriers, cannibales et prédateurs) que selon une logique de circulation interne (plus caractéristique des systèmes pacifiques, comme ceux du Haut-Xingu ou du Haut Rio Negro). La contribution principale de mon travail a été de formuler une hypothèse sur l'articulation entre ces deux modes d'action, simultanément présents dans la reproduction sociale kayapo (Gordon 2006). L'analyse du système rituel kayapo a fait émerger une distinction entre ses deux composantes dont les sens sont inversés et complémentaires : a) la transmission intergénérationnelle des prérogatives, objets et droits cérémoniaux ; b) la confirmation rituelle fonctionnant comme mécanisme de base de re-subjectivation et ayant une dimension sacrificielle. Les objets obtenus de l'étranger sont, au moyen de processus de métamorphoses rituelles, symboliquement reconnectés aux subjectivités différenciatrices et régénératrices de ses propriétaires originaux. Ils acquièrent ainsi une qualité extraordinaire (codifiée dans le concept indigène d'esthétique et de moral : « beau/beauté ») qui sera disséminée en interne grâce à la transmission intergénérationnelle.

La transmission intergénérationnelle et le sacrifice rituel des objets (et d'autres prérogatives) doivent être vus tous les deux comme des mouvements inhérents au régime de reproduction sociale kayapo. Le premier est associé à la production de l'identité et à la constitution d'une corporalité et d'une moralité spécifiques, qui doivent être partagées et reconnues collectivement, établissant les limites internes du mode de vie kayapo. Le second est associé à un processus de différenciation et d'affirmation de l'extraordinaire, de l'exceptionnel, du beau et du pouvoir qui, par définition, établit les limites externes de la vie kayapo et fait que le fons e origo de la beauté se trouve à l'extérieur. 


\section{LE CONTEXTE : LeS XIKRIN du CATETÉ ET L’ARgENT}

Les Xikrin du Cateté sont un des sous-groupes kayapo (auto-dénommé Mebêngôkre) qui vivent dans la région située entre les fleuves Tocantins et Araguaia, dans l'État du Para. Ils forment une population d'environ 1000 personnes, dont $80 \%$ ont moins de trente ans. Leur territoire comprend environ 400000 ha et se trouve dans le département de Parauapebas.

Actuellement, les Xikrin ont une relation particulière avec l'argent et les biens industriels qu'à défaut d'un autre terme plus approprié j'ai nommée relation de "consommation inflationniste ${ }^{4}$ », c'est-à-dire qu'ils expriment une demande toujours croissante pour de l'argent et pour des biens industrialisés. L'impression ressentie par le visiteur qui arrive dans leurs villages est qu'il y a une surabondance d'objets dans les relations entre les personnes, et les Indiens apparaissent comme des matérialistes ou des consommateurs frénétiques/consuméristes. Les biens industrialisés sont partout, en relation avec toutes les activités quotidiennes. Les Indiens ne s'intéressent pas qu'aux instruments et aux outils liés aux activités de subsistance. Au contraire, il y a un grand attrait pour les biens que nous avons l'habitude de considérer comme relevant d'un certain niveau de vie (sodas, vêtements et accessoires, réfrigérateurs, appareils électroniques, antennes paraboliques, lecteurs de DVD, voitures...).

Ce désir de biens industriels semble ne jamais être satisfait et s'apparente à une boulimie d'objets importés. Entre 1998, année durant laquelle j'ai été pour la première fois chez les Xikrin, et 2005, date de ma visite la plus récente, les changements de mode de consommation ont été impressionnants. En 1998, il n'y avait que très peu de maisons en brique, pas d'électricité et aucune voiture. En 2005, toutes les maisons étaient faites de briques, le village avait été entièrement raccordé à un réseau électrique et une dizaine de véhicules y circulait. Il est habituel de voir stationner à côté de la maison des hommes, au centre du village, une brillante voiture rouge appartenant au fils du chef, avec des vitres teintées et des jantes en alliage. L'argent est présent dans tous les esprits. Nombre de conversations dans la maison des hommes ou dans l'environnement domestique de la périphérie des maisons tournent inlassablement autour de l'argent (piokkaprin ${ }^{5}$ ). Tant au niveau individuel que collectif, les Xikrin utilisent beaucoup de temps et d'énergie pour élaborer des stratégies afin d'augmenter leur pouvoir d'acquisition et de consommation de biens manufacturés.

Notons que cette situation s'inscrit dans un long investissement historique des Kayapo envers les objets des Blancs. Un recensement historique permet d'affirmer que de tels objets étaient déjà une question importante pour eux bien avant l'établissement de contacts pacifiques définitifs avec la société brésilienne, qui ont eu lieu autour de 1950. Cette attirance pour les objets manufacturés a orienté les actions des Indiens, fonctionnant comme un catalyseur de la dynamique politique 
interne et externe des activités guerrières et des processus de reconfigurations des groupes depuis le fin du XIX ${ }^{\mathrm{e}}$ siècle.

L'ambition d'avoir son propre argent et d'obtenir des marchandises, hormis les spécificités locales, est apparue de manière similaire dans pratiquement tous les groupes kayapo. Dans les années 1980 et 1990, ces Indiens sont devenus célèbres par leur forte mobilisation pour obtenir des droits politiques et la démarcation de leurs terres, mais aussi en raison de la forme intensive avec laquelle ils entraient en relation avec les marchés locaux pour rechercher des biens industriels et des ressources monétaires. Leurs connexions avec les industries forestières et l'orpaillage sont connues, ainsi que l'apparition de leaders prestigieux ou enrichis, comme Raoni, Paulinho Pajakãn, Coronel Tyt-Pombo - phénomènes largement commentés dans la presse nationale et étrangère (Rabben 1998 ; Freire 2001) et thématisés par quelques ethnologues (Turner 1993, 1995 ; Fisher 2000).

Mais la situation des Xikrin, dans cet univers kayapo, est spécifique, en raison d'une conjoncture historique et géographique particulière : ils habitent un territoire contigu à la Serra dos Carajás, région où a lieu une des plus grandes exploitations minières du monde, le programme Grande Carajás, par l'entreprise multinationale brésilienne, Companhia Vale do Rio Doce (désormais Vale). Les droits d'exploitation du minerai ${ }^{6}$ dans cette zone, voisine du territoire xikrin, ont été conditionnés par une série de mesures compensatoires de l'impact des opérations industrielles, imposée par le gouvernement brésilien. Ainsi, depuis la fin des années 1980, Vale a négocié avec la Fundação Nacional do Índio (FUNAI) un contrat d'assistance aux Xikrin. Ce contrat a progressivement garanti une affluence importante d'argent et de marchandises directement vers la communauté, ainsi que la maintenance du poste indigène de la FUNAI et la mise en place d'infrastructures (construction de routes, de maisons, installation d'un générateur), en plus des services de santé, d'éducation et de transport. Aujourd'hui, pratiquement toutes les ressources dont ils disposent proviennent de leur lien avec Vale?

Le volume des ressources et leurs destinations sont décidés lors de réunions de programmation annuelles entre des représentants de Vale et des Xikrin. Ce sont des occasions où les Indiens se sont immiscés dans les questions politiques, exerçant une forte pression sur la grande entreprise, avec l'objectif de voir leurs demandes satisfaites. Ailleurs, j'ai démontré que les formes d'engagement des Xikrin lors de ces réunions politiques pouvaient être analysées en termes de logique guerrière et prédatrice. Même s'il n'y a pas nécessairement de violence réelle, du point de vue symbolique, tout se passe comme si les Xikrin adoptaient une perspective de sujet de la prédation, déplaçant les représentants de Vale (et métonymiquement tous les Blancs) vers la position d'objet (Gordon 2006 ; 2009).

Or, le fait de pouvoir compter sur des ressources financières régulières et virtuellement infinies rend le cas xikrin particulièrement intéressant dans l'univers kayapo. En effet, cela amplifie et rend plus visibles encore les processus qui 
sont à l'œuvre dans toutes les autres communautés kayapo, mais avec une vitesse et des degrés différents. Grâce au contrat avec Vale, les Xikrin ont élaboré des mécanismes pour institutionnaliser l'acquisition d'argent et de marchandises. À côté des ressources allouées aux programmes d'assistance a été créée une rente fixe, payée mensuellement aux Indiens, destinée à la consommation de biens non durables et nommée verba mensal (rente mensuelle). Avec ces ressources financières, les Xikrin ont organisé un système de répartition de l'argent et de distribution des marchandises acquises, ainsi qu'un système de salaire.

\section{LA POLITIQUE ET LES SALAIRES}

Un tel système de répartition et de distribution, défini par les Indiens euxmêmes, nous apprend énormément sur l'organisation politique et la structure hiérarchique de prestige des villages xikrin. Mais les Xikrin ont surtout réussi à augmenter la valeur de la rente mensuelle au cours des dernières années ce qui a eu pour effet une grande variation des salaires et leur extension. Cet aspect nous intéresse tout particulièrement car il reflète le caractère inflationniste dont j'ai parlé plus haut. Voyons brièvement comment ont été institués les salaires des chefs.

Au début des années 1990, après le déclin des affaires avec les exploitants forestiers, les chefs, qui en étaient les principaux bénéficiaires, ont commencé à discuter avec le personnel de Vale, proposant l'implantation d'une politique salariale dans le cadre du contrat. Il s'agissait d'une espèce de compensation liée aux responsabilités des chefs qu'ils présentèrent comme harassantes et d'une tentative de disposer de ressources personnelles, vu que la fin des relations avec les exploitants forestiers avait réduit le contrôle qu'ils exerçaient sur l'argent, les marchandises et les services. Ainsi, tout en étant considérée comme l'argent de la communauté, une partie de la rente mensuelle a été utilisée pour verser des salaires aux chefs.

À ce moment-là, il y avait deux villages, chacun avec son propre chef. Ceux-ci étaient les fils de deux frères, eux-mêmes anciens chefs de village. Initialement, seuls ces quatre chefs principaux (c'est-à-dire les deux chefs de villages et leur père respectif, ex-chef de village) reçurent une somme d'argent, représentant un volume d'environ $10 \%$ de la rente mensuelle. Ces chefs utilisèrent par la suite une partie de leur rétribution personnelle pour rémunérer des secrétaires ou assistants. Rapidement, ils se sont toutefois aperçus qu'ils pouvaient amplifier le système salarial. Après quelques réunions, les chefs mineurs (c'est-à-dire les leaders de groupes d'âge, ou turma comme disent les Xikrin en portugais) ont aussi obtenu leur propre salaire. En 1998, il y avait sept hommes salariés.

La valeur de la rente mensuelle était cependant considérée comme insuffisante par les Indiens. La valeur de la rente fut alors l'objet d'intenses négociations de la part des chefs mineurs pour que les demandes en marchandises de leur groupe 
puissent être couvertes. Ces négociations furent à l'origine de tensions importantes entre les chefs de groupes, ainsi qu'à l'intérieur de chacun des groupes. C'était au moyen de la médiation des chefs que l'argent de la rente mensuelle revenait à la communauté sous la forme de biens, chaque chef mineur étant responsable de la distribution de marchandises entre les membres de son groupe, au moyen de l'élaboration d'une liste d'achats.

En 1999, après de dures négociations avec les représentants de Vale, durant lesquelles les Xikrin menacèrent d'envahir le siège de l'entreprise et de paralyser les opérations industrielles de la mine de Carajás, ils réussirent à obtenir le doublement de la rente mensuelle. Les salaires des chefs principaux connurent alors une augmentation dans les mêmes proportions (100\%). La fiche de paie a touché d'autres leaders. De sept, les leaders salariés sont en effet passés à dix.

Néanmoins, cette promotion salariale a rapidement été jugée insuffisante. Au début de l'année 2001, une nouvelle réunion de programmation eut lieu et, une nouvelle fois, Vale a été contrainte, sous la pression des Indiens, à réajuster la valeur de la rente mensuelle. En ce qui concerne les salaires, la tendance expansive s'est maintenue dans les deux sens : 1) augmentation pour les chefs et les leaders qui en étaient déjà bénéficiaires ; 2) extension du statut de salarié à de nouveaux individus, presque toujours liés aux chefs, par consanguinité ou affinité. De dix, les salariés sont passés à quinze.

Et ce processus semble devoir continuer indéfiniment ${ }^{8}$. À chaque augmentation, la situation se calme pour un temps, puis rapidement surgit la nécessité d'une nouvelle augmentation. La Figure 1 récapitule l'évolution de la valeur de la rente mensuelle entre 1998 et 2005 . Il fournit une bonne image de l'augmentation de plus de $1000 \%$ qui a eu lieu en 6 ou 7 ans! À titre de comparaison, on peut aussi regarder l'évolution des salaires des chefs de village sur la même période (Figure 2).

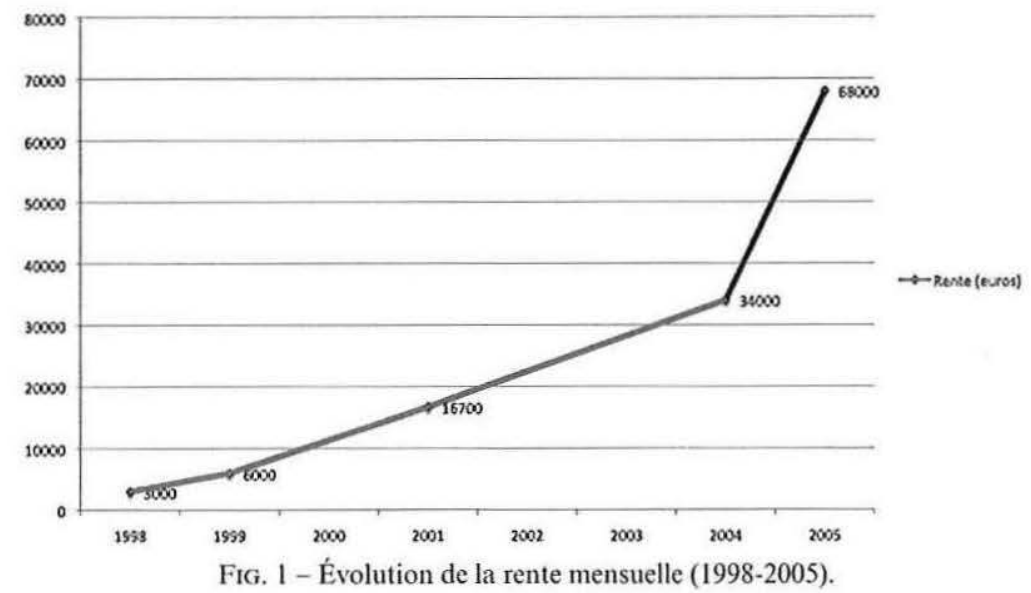




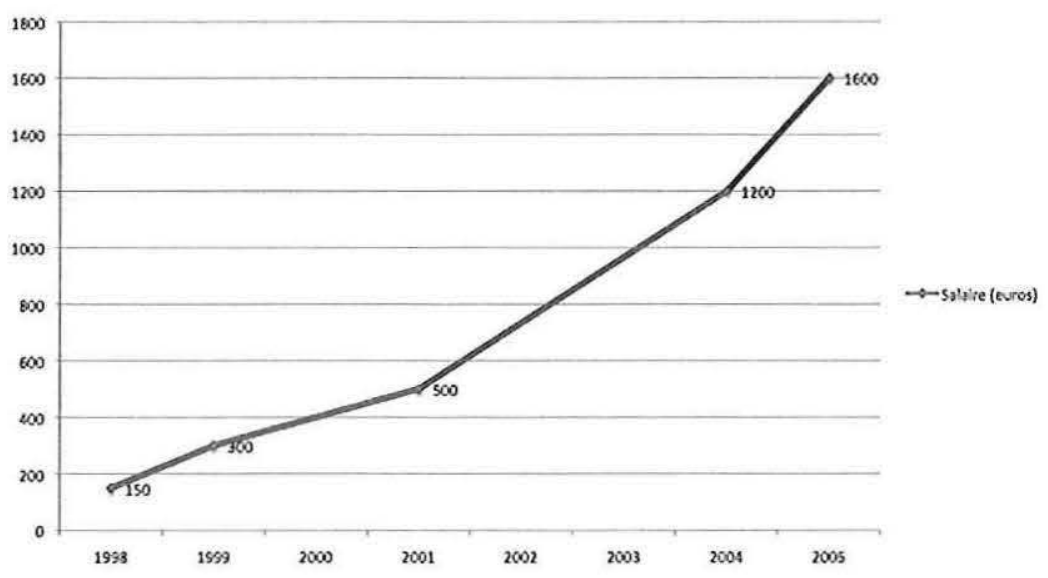

FIG. 2 - Évolution du salaire des chefs (1998-2005).

La hiérarchie entre les leaderships est marquée par la valeur des salaires. Les chefs principaux de chaque village reçoivent les plus hauts salaires $(1600 €)$. Ils sont suivis par les chefs de groupes d'âge ou turma (1200€), puis par les simples leaders $(1000 €)$, enfin par un groupe de jeunes leaders ayant des salaires moindres. À date récente, au moment de la dernière augmentation de la rente mensuelle, les chefs ont décidé que tous les hommes mariés de chaque village recevraient aussi de l'argent, soit une somme que l'on peut estimer à 100 ou $150 €$. On le constate, il existe une concentration significative des ressources entre les mains des chefs, des leaders et de leurs familles. En vérité, l'analyse généalogique permet de montrer qu'il s'agit d'une même parentèle (bilatérale), très prestigieuse et dotée d'un grand pouvoir politique.

Dans la Figure 3 sont numérotés de 1 à 15 tous les individus qui recevaient un salaire provenant de la rente mensuelle jusqu'en $2005^{9}$. Ce sont des chefs majeurs de village (1, 2, 3 et 4), des chefs mineurs ou leaders de classes d'âge masculines $(6,9)$, des leaders ou assistants $(5,7,8,10,11,15)$ et des jeunes leaders qui font leur début comme assistants (12, 13 et 14).

En observant la Figure 3, on voit clairement comment les principales positions de leadership et de prestige chez les Xikrin - exprimées actuellement au moyen de l'accès différentiel à l'argent et aux marchandises - sont concentrées et transmises à l'intérieur d'un même groupe de parents, reliés entre eux bilatéralement. De plus, les autres positions de chef et de leader semblent graviter autour de ce noyau. Tous ceux qui entrent dans le processus de la feuille salariale de la rente mensuelle sont d'une certaine manière liés (par des liens immédiatement identifiables) à des personnes qui ont occupé une position de leader et se sont distinguées dans le passé. 


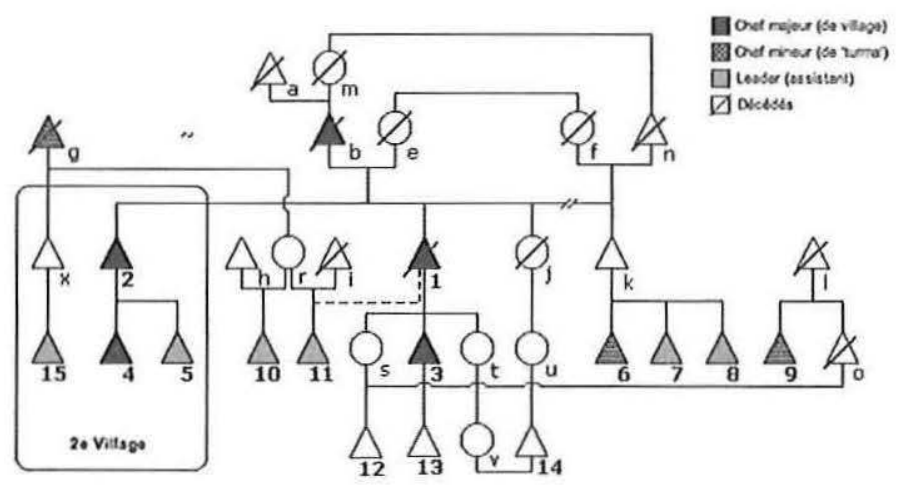

FIG. 3 - Individus (numérotés) recevant un salaire.

La différence d'accès aux ressources entre chefs et non-chefs est donc une caractéristique générale de la façon dont les Kayapo appréhendent les marchandises (Fisher 2000 ; Turner 1993 qui décrit le phénomène comme étant l'apparition d'une hiérarchie de classes dans la société Kayapo ${ }^{10}$ ). De manière plus incisive et expressive, cette différence apparaît dans le discours de nombreux Xikrin, notamment des non-chefs qui répétaient sans cesse : « le chef est riche, la communauté est pauvre».

Mais cette pauvreté est relative, car le système de division des ressources garantit un niveau raisonnable de consommation pour tous les Xikrin. Cela dit, par ce type d'affirmation c'est un principe de différenciation très clair qui est exprimé. Devant ces données, deux questions principales s'imposent, auxquelles je vais tenter de répondre : 1) Cette différence dans l'accès aux ressources est-elle un phénomène totalement nouveau dans la société kayapo, une espèce d'intrusion d'une logique capitaliste dans une société jusqu'alors « indivise », pour reprendre une expression chère à Clastres (1974), ou résulte-t-elle de la transformation d'un processus de différenciation préexistant entre les Kayapo ? 2) Comment expliquer le caractère inflationniste? Autrement dit, pourquoi ne semblet-il pas y avoir de limites aux demandes des Xikrin concernant l'argent et les marchandises, qui augmentent de manière exponentielle?

\section{LES MARCHANDISES}

Il convient de préciser d'emblée que les Xikrin n'accumulent pas l'argent qu'ils obtiennent, mais qu'ils le convertissent rapidement en biens et marchandises qui sont consommés dans la vie quotidienne et lors des périodes cérémonielles. L'argent est perçu localement comme un instrument de transformation par 
excellence, un moyen d'acquérir pacifiquement tous les objets désirés, objets qui sont produits uniquement et possédés par les Blancs. L'argent est donc, pour les Xikrin, une sorte de capacité à capturer les objets étrangers (capacité qui est, elle-même, une émanation de l'étranger et doit aussi être capturée).

Cette question de la capture d'éléments étrangers nous renvoie directement à l'univers rituel kayapo. C'est en effet justement dans le contexte cérémoniel que nous rencontrons un ensemble de biens de valeur symbolique, tous originaires de l'extérieur; dont l'effet de différenciation est parallèle à l'actuelle séparation riches/pauvres. Je me réfère aux biens cérémoniels - noms personnels et prérogatives (nêkrêjx) - qui ont été décrits par Lea (1986) comme la richesse de la société traditionnelle kayapo. La dynamique de transmission et de ritualisation de ces biens est à la base d'une distinction entre belles personnes et personnes communes. Tout mon travail a été de montrer que ces deux modes de distinction (riches/pauvres ; beaux/communs) forment un seul et même processus, perçu à des moments différents de l'histoire kayapo.

L'association entre prérogatives rituelles (nêkrêjx) et marchandises a d'abord été pointée par les Indiens eux-mêmes, puis par les ethnologues (Lea 1986, p. 348 ; Turner 1993, p. 63). Initialement, le terme kayapo pour marchandise était nêkrêjx. Les premiers objets industrialisés que les Kayapo ont obtenus des Blancs - que ce fût par troc ou pillage - ont été incorporés en tant que biens cérémoniels. On trouve, de fait, mention, chez les Xikrin et dans d'autres groupes kayapo, d'objets industrialisés et d'autres biens provenant des Blancs qui sont effectivement entrés dans le système traditionnel de transmission des biens cérémoniels. Je crois que, dans tous ces cas, il s'agissait d'objets obtenus avant l'établissement de contacts pacifiques avec l'État brésilien et l'intensification de la relation mutuelle, période pendant laquelle les Xikrin et les Kayapo avaient moins accès aux objets industrialisés qu'aujourd'hui. On peut prendre, pour exemple, le chapeau rouge signalé par Lea (1986, pp. 347-348) chez les Kayapo, ou les robes exhibées comme trophées par les Mekranoti (Verswijver 1992, p. 146) et d'autres objets comme le parapluie, les tasses en verre, la pagaie et, même, des animaux comme le chien et la poule (Gordon 2006, pp. 357-359).

Bien que les récits historiques et ethnographiques ne soient pas concluants sur cette question, il n'est pas complètement impossible que, de par leur rareté et leur nature considérée comme exotique, les premiers objets des Blancs que les Kayapo se sont appropriés - y compris les objets que l'on pourrait appeler utilitaires comme les machettes, les haches et les fusils - aient été, d'une certaine manière, traités comme des biens cérémoniels. Un homme xikrin m’a déclaré que, dans un passé lointain, certains conservaient les fusils dont ils s'étaient emparés lors de raids effectués chez les Blancs, et ce, même s'ils ne disposaient pas de munitions, dans le but de les porter lors de certaines danses cérémonielles. Le fait est qu'après un certain temps, nombreux sont ces objets qui ont cessé d'être traités comme des prérogatives rituelles personnelles, n'étant plus transmis selon la règle d'héritage. 
En d'autres termes, ces objets sont passés par un processus de «communisation " ${ }^{11}$ au cours duquel leur valeur utilitaire est venue se superposer à leur valeur cérémonielle, élargissant leur circulation, voire permettant à tous les membres de la communauté un accès direct à ceux-ci. Comme l'ont bien montré Verswijver (1992) et Lea (1986), on pourrait toutefois se trouver face à des situations moins nettes dans lesquelles une marchandise, utilisée sans restriction dans des contextes non cérémoniels, deviendrait restrictive dans des contextes rituels. Laissons de côté les cas sujets à controverses, dans lesquels l'usage (cérémoniel ou non) était parfois contesté par des individus ou des familles.

En définitive, ma réponse à la première question posée plus haut est qu'il existe une continuité. Mais il ne s'agit pas d'une continuité simple et directe, parce qu'aujourd'hui les marchandises ne sont pas exactement comme des nêkrêjx. De ce fait, les marchandises sont, pour la plupart, canalisées par les chefs, distribuées dans un système théoriquement universel et qui s'appuie sur les organisations collectives, les turmas. Elles peuvent être utilisées par les Xikrin de manière équivalente à la nourriture, en circulant entre diverses catégories de parents et d'affins. D'autres objets, dont la consommation est plus restreinte ou différentielle, ne sont pas non plus attribués à la manière des prérogatives, mais ont tout de même tendance à se concentrer dans les mains de certaines familles, cognatiquement définies et liées par l'alliance. De nombreuses marchandises sont finalement présentes dans la vie cérémonielle, mais principalement sous la forme d'aliments rituels ou de matière première pour la fabrication d'ornements.

Où se trouve alors la continuité ? Pour pouvoir postuler cette continuité de manière plus consistante, il faut que nous revenions maintenant aux biens cérémoniels, à leurs circulations et leurs effets dans l'économie de valeur kayapo. Il faut mettre au jour complètement le fonctionnement du système des biens cérémoniels et la logique qui se cache derrière celui-ci.

\section{LES BEAUX ET LES COMMUNS, LES RICHES ET LES PAUVRES}

Les ensembles de biens symboliques que les groupes kayapo possèdent traditionnellement incluent des noms personnels (appelés "grands » ou " beaux noms ») et des prérogatives cérémonielles (nêkrêjx) qui fonctionnent comme signes distinctifs individuels et collectifs. De tels biens sont à la base d'un mécanisme de production de valeur, exprimé par le concept xikrin de " beauté » (mejx) ${ }^{12}$.

Les Kayapo font une distinction entre les personnes porteuses de ce qu'ils appellent les « beaux noms » (idji mejx), nommées me mejx - personnes bonnes ou belles (parfaites ou complètes) - et celles dotées de "noms communs » (idji kakrit), nommées me kakrit - personnes communes (imparfaites ou incomplètes). Noms et prérogatives sont la propriété de certaines personnes ou certaines 
familles et sont transmis d'individu à individu, d'une génération à l'autre, au moyen d'une règle fixe qui fait intervenir les parents croisés ${ }^{13}$.

Un aspect de la puissance et de la beauté des noms provient de leur origine externe et animale, ainsi qu'on peut le déduire de la mythologie. Pour les Kayapo, ces noms proviennent d'êtres surnaturels associés au domaine aquatique (poissons mythiques). Les Kayapo auraient obtenu ces noms grâce aux héros culturels mythiques qui les ont volés aux poissons. De la même manière, la puissance et la beauté des prérogatives, savoirs et objets rituels, proviennent aussi de leur origine externe et de leur exclusivité ou rareté. De nombreux ornements de plumes, objets cérémoniels, chants et même des cérémonies entières ont été pris par les Kayapo à d'autres peuples, étrangers, au cours de leur histoire. En outre, l'origine des ornements à plumes est pensée et exprimée dans les mythes comme le résultat d'une action prédatrice des Indiens contre un gigantesque oiseau surnaturel et cannibale.

Il est possible que l'ensemble des noms et prérogatives ait constitué dans le passé kayapo un système de type clanico-totémique, tel que nous le connaissons dans d'autres groupes de langue ge et bororo. Dans le cas des Kayapo, noms et prérogatives, comme le montre très bien Lea (1986), sont devenus objets de dispute du fait d'avoir constitué une richesse symbolique. Familles et individus tentent d'accumuler ces biens, contrôlant de manière rigide le mécanisme de leur transmission au moyen de prêts et de dévolutions, de façon à éviter une dispersion de l'objet ou du nom. Il est important de pouvoir retracer la ligne généalogique de circulation d'un bien, depuis son origine jusqu'au présent. Mais, évidemment, ce n'est pas toujours simple car il y a régulièrement des disputes et des accusations de vol de noms et de prérogatives.

Le fait que l'origine exogène doit être réactualisée rituellement pour garantir l'effet de valeur et de beauté des noms et prérogatives est également un point fondamental. La transmission générationnelle top-down ne garantit pas, à elle seule, la valeur des noms et prérogatives rituelles. Elle peut, au contraire, produire leur dévalorisation. En revanche, la confirmation rituelle, qui lie les objets cérémoniels aux propriétaires originels dans un sens bottom-up, est ce qui garantit leur vraie valeur et leur vraie beauté. Un objectif des grands rituels kayapo est de vérifier et de confirmer, publiquement et collectivement, les noms et les prérogatives cérémonielles transmis aux enfants par leurs donneurs de noms. Les enfants à qui l'on rend hommage ou qui sont honorés durant les rituels sont nommés mereremejx, expression qui a un sens proche de " ceux à qui se donne/se concède la beauté », " ceux qui deviennent beaux ». Les parents des enfants célébrés sont dénommés mekrareremejx, c'est-à-dire "ceux dont les enfants sont devenus beaux ». L'importance de la connexion cérémonielle entre noms et prérogatives pour l'obtention de cette qualité est par ailleurs assez claire : les noms sont dits kajgo, c'est-à-dire «inutilement beaux, faussement beaux » - c'est-à-dire sans véritable effet donneur de valeur ou de beauté -, du moins tant qu'ils n'ont pas été 
confirmés comme tels au cours d'une cérémonie en adéquation avec cet objectif (chaque ensemble de noms est associé à un type de rituel spécifique). Par une série de métamorphoses rituelles - quand les Kayapo se transforment en Autre -, les objets cerémoniels sont symboliquement reconnectés aux propriétaires originaux et conquièrent une nouvelle puissance différenciatrice ou régénératrice. Le rituel, de ce fait, a pour conséquence une re-subjectivation de ces biens, qui leur confère une sorte de valeur sacrée. Pour utiliser une analogie, les cérémonies rituelles fonctionnent un peu comme un chargeur de batteries. L'objet (nom ou prérogative) est là, mais comme une pile vide, il est inerte avant d'être rechargé en énergie. Ainsi, il ne suffit pas à un enfant d'avoir reçu les noms et prérogatives de parents déterminés. Il faut que ces biens soient confirmés rituellement.

Les rituels kayapo, même s'ils sont collectifs, possèdent un caractère privé. Ils doivent être promus par les parents et les proches des enfants. Les parents doivent être capables de créer les conditions matérielles qui permettront la réalisation de la cérémonie. D'une certaine manière, les parents payent en nourriture pour que les autres personnes du village dansent et participent à la fête. Ainsi, les familles déploient toujours de grands efforts pour promouvoir les cérémonies de nomination de leurs enfants, arrangeant certaines relations de parenté avec l'idée de fournir l'aliment rituel nécessaire à l'organisation des fêtes de façon à garantir aux enfants la condition de me mejx. Le problème est que tous n'arrivent pas à le faire.

Le système cérémoniel crée donc in fine une division interne, exprimée explicitement dans le discours des Indiens : la différence passe entre belles personnes ( $m e$ mejx) et personnes communes (me kakrit). Cette différence a aussi une dimension politique, vu qu'elle est associée aux relations sociales qui sont à la base du choix des positions de leadership.

Si la confirmation rituelle recharge en énergie les batteries (les objets) en leur conférant une valeur exceptionnelle, la transmission générationnelle peut les décharger. La circulation, si elle est excessive, peut mener à un effet inverse de la re-subjectivation rituelle, c'est-à-dire qu'elle cause une dévalorisation et une objectivation d'un bien. Objectivé, il devient quelque chose de commun, qui peut être d'un usage généralisé dans le groupe, mais devient nettement moins intéressant pour les personnes et les familles qui recherchent prestige et distinction. Pour que ces objets soient capables de procurer beauté et prestige, il faut simultanément contrôler leur transmission (en évitant qu'ils se répandent trop, en se dévalorisant et devenant de simples objets) et garantir leur re-subjectivation rituelle. Ce jeu qui produit de la valeur, mais en même temps donne des limites à une possible valorisation, procure au système une dimension dynamique et ouverte. Historiquement, cela se reflète dans le caractère centrifuge de la société kayapo qui a toujours pratiqué la guerre avec, comme objectif, l'obtention de nouveaux éléments pour son économie politique de valeur et de beauté.

Quand les Blancs ont fait irruption dans la vie kayapo, porteurs d'une quantité fascinante de nouveautés et d'objets, ces derniers ont immédiatement 
fait l'objet d'un intérêt particulier, motifs de capture et de rénovation de ce qu'on pourrait appeler des "stocks de distinctions », une forme de production de la distinction. Les marchandises sont devenues, de nouveau, nêkrêjx.

Nous assistons, aujourd'hui, pour ce qui est du traitement des marchandises et de l'argent par les Xikrin, à un mouvement qui, je crois, a toujours existé en ce qui concerne les objets cérémoniels. Ce mouvement est lié à la façon dont les Kayapo conçoivent leur relation à l'altérité et dont ils traitent rituellement les éléments qui incarnent cette relation. Il existe, toutefois, quelques différences importantes qu'il ne faut pas minimiser. Pour commencer, on observera que la plus grande part des marchandises qui circulent aujourd'hui dans le quotidien xikrin n'est pas liée à la sphère cérémonielle et au système de transmission verticale. Comme je l'ai dit, il y a trop de marchandises et elles sont partout, ce qui empêche la limitation de leur circulation au moyen d'une règle d'héritage, comme dans le cas des noms et nêkrêjx.

Tel était, pourtant, le cas dans le passé. Les premiers biens industriels obtenus par les Xikrin et les Kayapo ont été traités exactement comme les nêkrêjx: devenant des prérogatives de certaines personnes et de certaines familles, ils étaient transmis par la règle de parenté mentionnée plus haut. Mais, face au développement de l'histoire et à l'intensification des relations avec la société brésilienne, l'accumulation et la quantité croissante de biens industriels disponibles (nombre d'entre eux utilitaires et dépassant l'usage rituel exclusif) ont eu pour effet de modifier leur mode de circulation. Chez les Xikrin, nous l'avons vu, l'argent et les marchandises ont commencé à circuler à partir du système de groupes politiques masculins, système prolongé par des mécanismes de distribution horizontale et générale. Pourtant, cette façon, plus ou moins universelle, de distribution n'exclut pas une tendance certaine à la concentration et à l'exclusivité, comme si, même en dehors du système de distinction rituelle, les marchandises continuaient à pouvoir être utilisées pour créer (ou aider à composer) des différences internes de valeur, et c'est en cela qu'elles ont une fonction nêkrêjx. Jadis, la différence majeure était entre les belles personnes et les personnes communes. Aujourd'hui elle se situe entre celui qui a beaucoup et celui qui a peu d'argent et de biens. Les beaux sont devenus les riches.

\section{CONCLUSION : PARADOXE ET INFLATION}

Il nous est maintenant possible de revenir au problème de l'inflation xikrin en faisant la synthèse de ce qui a été dit.

Dans la dynamique sociopolitique et culturelle xikrin et kayapo, le mécanisme de production de valeur et de distinction - exprimé dans l'idée de beauté - était fondé sur un certain traitement de biens symboliques qui fonctionnaient comme des signes de relation avec l'extérieur, avec ce qui est défini par les Xikrin comme l'altérité. À la différence des autres biens considérés communs à tous et dont le 
partage est obligatoire (comme la nourriture, par exemple), ces biens symboliques obtenaient leur valeur distinctive au moyen de deux processus : une règle de transmission verticale - qui garantissait une circulation réduite identifiant avec une certaine clarté une ligne familiale - et un processus symbolique - que j'ai appelé « re-subjectivation » rituelle - qui re-potentialisait le caractère exogène et les capacités régénératives et différenciatrices de ces biens. Un tel système présentait une tendance dynamique, s'appuyant sur un jeu constant de valorisation et de dévalorisation lié à la forme selon laquelle ces biens pouvaient devenir plus ou moins communs, d'accès plus ou moins réduit à toute la communauté.

L'entrée des objets et des valeurs du monde capitaliste industriel - même si elle a été promue par les Xikrin, pour leurs propres finalités, et même si elle présente des continuités structurales - a amené quelques modifications importantes dans ce système. En sortant du cadre traditionnel de traitement (transmission verticale et connexion rituelle re-subjectivante), les marchandises ont constitué un certain paradoxe sociologique : elles sont devenues des objets devant circuler de manière à la fois restreinte (suivant la logique de distinction) et élargie (pour intensifier les liens communautaires et de parenté entre les personnes). Pour cela, elles ont causé une accélération de toute la dynamique, dans la mesure où la pression pour les rendre communs provoque de nouvelles tentatives de la part de plusieurs individus et familles pour les particulariser aux prétentions de leadership et de prestige - tentatives qui se concrétisent par la recherche de nouvelles formes de consommation et d'un accès à de plus grandes quantités de marchandises, mais qui sont à leur tour soumises à de nouvelles exigences de mise en commun et ainsi de suite.

Pour accentuer encore l'évolution, le développement des activités rituelles qui se vérifie à présent (résultat des nouvelles conditions technologiques et matérielles) semble promouvoir une mise en commun du statut cérémoniel de beauté, dissolvant la distinction entre belles personnes et personnes communes, et rendant commun le droit d'usage de nombreux objets rituels, auparavant vus comme exclusifs de certaines personnes et familles. Une enquête quantitative montre qu'historiquement la croissance démographique des trois dernières décennies a été accompagnée d'une intensification de l'activité rituelle chez les Xikrin (Gordon 2006, pp. 336-339). Il est important d'observer que les chefs contribuent, d'habitude avec leurs propres ressources, mais surtout avec leur rente mensuelle, à la logistique de la fête, allégeant ainsi le fardeau des familles qui, jadis, devaient déployer un énorme effort productif pour confirmer le statut cérémoniel de leurs enfants. De fait, dans le passé, des familles peu nombreuses ou sans connexions politiques importantes, ne réussissaient à confirmer cérémoniellement qu'un ou deux enfants ou, même, aucun. De nos jours, les chefs fonctionnent comme des sponsors de fêtes, occupant un rôle qui, jadis, incombait aux familles.

Le nombre d'enfants célébrés lors de fêtes de nomination commence à augmenter considérablement. Des portions de plus en plus grandes de la commu- 
nauté célèbrent leur nomination et sont considérées comme belles. Ainsi la différence beaux/communs, si souvent soulignée par les ethnographies, semble devenir sociologiquement moins importante, ou moins marquée, au profit d'une nouvelle formule : riches (ayant de l'argent)/pauvres (sans argent). Je ne veux pas dire par là que la distinction beaux/communs ait été supprimée. J'attire simplement l'attention sur une certaine délimitation du problème chez les Xikrin. Actuellement, ceux-ci semblent insister beaucoup moins sur cette différence que dans le passé. Par ailleurs, de nos jours, il semble y avoir beaucoup plus de discussions et de tensions autour du fait de savoir qui est riche ou pauvre, qui a ou n'a pas d'argent et un certain pouvoir d'achat.

Comme je l'ai déjà mentionné, l'argent n'est pas accumulé, mais il sert de moyen d'accès immédiat à de plus grandes quantités et variétés de marchandises. Néanmoins, dans la mesure où le système acquiert vitesse et accélération, la capacité de re-subjectiver et de valoriser les marchandises par la quantité ou la variété commence aussi à donner des signes d'épuisement : les augmentations successives de la rente mensuelle et le mécanisme organisé (et constant) de distribution garantissent un accès relativement universel aux marchandises dans la communauté, même s'il est différé dans le temps. Par exemple, les chefs ont été les premiers à acheter des télévisions, puis en peu de temps toutes les maisons du village en avaient une. De même, les chefs ont été les premiers à posséder des réfrigérateurs, puis en peu de temps ces appareils ont été présents dans toutes les familles. Les chefs ont eu besoin de découvrir de nouveaux biens et ont acquis des lecteurs DVD... Tout cela résulte d'une pression constante à augmenter le volume des ressources financières. D'où la dimension expansive ou inflationniste de l'économie xikrin.

Face à ce cadre de rapide dévaluation des éléments de distinction interne, la question des salaires, c'est-à-dire de l'argent, acquiert un aspect intéressant. Les Xikrin sont peut-être en train de s'apercevoir que l'argent vaut moins pour son pouvoir d'achat que pour sa capacité infinie de différenciation du moment que se maintient l'emphase sur sa nature quantitative. À la différence des objets concrets, l'argent est quelque chose qui peut augmenter indéfiniment en maintenant sa capacité distinctive, du moment que le rapport différentiel est maintenu. Pour plus de clarté, si, aujourd'hui, j'ai la quantité 10 et vous la quantité 1 , il n'y aura pas de problème si, demain, vous avez la quantité 10 et moi la quantité 100 , du moment que le rapport entre nos quantités se maintient. L'afflux croissant de ressources monétaires dans la société xikrin obéit à ce type de principe, comme cela peut se vérifier dans la structure salariale des chefs et des leaders scrupuleusement divisée en échelons. Augmenter indéfiniment la quantité d'argent semble être la solution que les Xikrin ont trouvée pour compenser la rapide perte de valeur différenciatrice de leurs objets. C'est de cette façon que l'on peut définir l'inflation xikrin.

Tout cela pose toutes sortes de questions dont l'analyse dépasse le cadre de cet article. On se contentera pour le moment d'indiquer que les Xikrin ont conscience 


\section{d'être confrontés à un paradoxe et savent qu'il leur faudra regarder simultané- ment vers le passé et vers le futur pour en trouver l'issue. *}

* Manuscrit reçu en août 2009, accepté pour publication en juillet 2010.

\section{NOTES}

Cet article est une version modifiée d'un travail que j'ai exposé au séminaire du Centre d'enseignement et de recherche en ethnologie amérindienne (EREA) du Laboratoire d'ethnologie et de sociologie comparative, organisé et dirigé par Jean-Pierre Chaumeil. Pour leurs invitations et commentaires, je remercie chaleureusement Jean-Pierre et Bonnie Chaumeil, Philippe Erikson, Cristiane Lasmar, Eduardo Brondizio, Emmanuel de Vienne et Isabelle Daillant. J'adresse également mes remerciements à Philippe Erikson et Romain Simenel pour leur lecture soignée et attentive qui m’a beaucoup aidé à améliorer ce texte. Je reste toutefois le seul responsable de mes prises de position. Ma reconnaissance va enfin à Philippe Descola pour m'avoir reçu au Laboratoire d'anthropologie sociale comme chercheur invité durant l'année 2008-2009. La recherche chez les Xikrin a été financée par la Financiadora de Estudos e Projetos (FINEP) et par la Wenner-Gren Foundation for Anthropological Research. La première version de ce texte a été traduite par Jérémy Déturche.

1. Ce nouvel angle d'approche ethnologique commence tout juste à être adopté de manière plus systématique, comme le montre un ensemble de monographies et de travaux récents sur certains de ces thèmes (Albert et Ramos 2002 ; Lasmar 2005; Kelly 2005; Andrello 2006 ; Calavia 2006; Gordon 2006 ; Vilaça 2006 ; Lima 2008; Capiberibe 2007; Bonilla 2009; Erikson 2009; Hugh-Jones 2009; Turner 2009). Les lacunes se comblent, mais il reste encore beaucoup à faire.

2. Au Brésil, une des premières ethnographies à mentionner l'incorporation de l'argent et des biens de consommation dans le quotidien des Indiens fut celle de Mindlin (1985) chez les Surui, groupe tupi du Rondônia. Avant elle, Hoffmann (1964) avait publié un travail qui associe argent et acculturation chez les Shipibo (je remercie Philippe Erikson pour m'avoir signalé cette référence). Voir aussi HughJones (1992), Albert (1988; 1993), Overing (1992), Turner (1992), Fisher (2000) et Howard (2002).

3. Dans un autre travail (Gordon 2006, pp. 95-105), j'ai tenté de démontrer que cette inflexion différente se voyait également exprimée par le poids relatif octroyé à la question de la mort dans les régimes prédatoires tupi et ge. Je suggère que, pour les Ge ou du moins dans le cas des Kayapo, la destruction physique ou la déconstitution corporelle de l'ennemi est sociologiquement et symboliquement moins productive que le vol de biens culturels (tels que le feu, les noms, les chants, les ornements). $\mathrm{Et}$, de fait, les thèmes du raid, du pillage et du vol sont saillants dans un ensemble de mythes et de récits ge (Wilbert 1978; voir également l'excellent essai de Seeger 1993, sur l'assaut donné par les Suya à l'expédition de Karl Von den Steinen).

4. L'utilisation que je fais de l'expression « inflation » pour aborder l'économie xikrin n'est pas à proprement parler l'usage courant dans la théorie économique - où il indique grosso modo la chute de la valeur de marché ou le déclin du pouvoir d'achat de l'argent. Je l'utilise pour dire que, dans l'économie xikrin, existe une demande toujours croissante et, virtuellement, infinie pour l'argent et les biens, qui semble en relation avec une perte relative de valeur de certains biens cérémoniels traditionnels.

5. C'est un néologisme de la langue kayapo pour désigner l'argent : piok $\approx$ feuille, papier ; kaprin $\approx$ vieille, pâle, chagrin.

6. Il s'agit de l'exploitation d'un grand nombre de minerais (cuivre, or, manganèse, bauxite, nickel, cassitérite) dont le plus important est le fer.

7. Les Xikrin ont aussi connu une période durant laquelle ils se sont engagés dans des activités d'extraction de bois précieux, jusqu'au début des années 1990. Mais cette activité a été contenue suite à l'intervention d'anthropologues et d'ONG et grâce au contrat avec Vale.

8. En réalité, il a connu une interruption en 2007 à cause de problèmes relationnels entre les Xikrin et Vale. Après un incident survenu à la fin de l'année 2006, quand un groupe d'Indiens armés a envahi 
le Serra de Carajá, Vale décida de suspendre le contrat. Le Ministère publique et la FUNAI intentèrent une action judiciaire et le conflit fut transféré à un tribunal. Le juge rendit une sentence à la fin de l'année 2008 qui obligeait Vale à reprendre les paiements et demandait une étude afin de revoir leurs valeurs. Pendant ce temps, la FUNAI - et non les Indiens directement - était devenue responsable de la gestion de l'argent.

9. Aujourd'hui la situation est différente, mais je n'ai pas de données actualisées.

10. Voir Werner $(1981 ; 1982)$ pour une discussion sur la transmission héréditaire du leadership chez les Kayapo comme un effet du contact.

11. J'utilise ce néologisme pour définir le processus selon lequel des objets sont perçus comme étant une propriété collective et non particulière de certains (individus ou familles).

12. Le champ sémantique du mot mejx couvre une série d'attributs moraux et esthétiques que nous pourrions caractériser par les termes : bon, beau, correct, parfait, optimal.

13. Un tel mécanisme de circulation verticale est très bien documenté dans l'ethnographie des peuples ge, et il suffit ici de le remémorer brièvement. Un garçon reçoit noms et prérogatives d'un ou plusieurs parents masculins, de la catégorie ngêt (incluant ses MB, MF, FF...). Une fille reçoit noms et prérogatives d'une ou plusieurs parentes féminines, de la catégorie kwatyj (incluant FZ, MM, FM...). Par rapport à ces parents, Ego des deux sexes - c'est-à-dire l'individu qui reçoit - est dans la catégorie tàbdjwö.

\section{RÉFÉRENCES CITÉES}

\section{Albert Bruce}

1985 Temps du sang, temps des cendres : représentation de la maladie, système rituel et espace politique chez les Yanomami du sud-est (Amazonie brésilienne), thèse de doctorat, Université Paris X, Nanterre.

1988 «La fumée du métal : histoire et représentations du contact chez les Yanomami (Brésil) », L'Homme, 28 (106-107), pp. 87-119.

1993 «L'or cannibale et la chute du ciel : une critique chamanique de l'économie politique de la nature (Yanomami, Brésil) », L'Homme, 33 (126-128), pp. 349-378.

1997 «Etnographic situation' and ethnic movements: notes on a postmalinowskian fieldwork ", Critique of anthropology, 17 (1), pp. 53-65.

Albert Bruce et Alcida Ramos (éds)

2002 Pacificando o branco. Cosmologias do contato no norte-amazonico, UnespImprensa Oficial do Estado, São Paulo.

\section{ANDRELLO Geraldo}

2006 Cidade do Indio. Transformações e cotidiano em Iauarete, EdUnesp/Isa/Nuti, São Paulo.

\section{Bonilla Lydie Oiara}

2009 "The skin of history : Paumari perspectives on conversion and transformation ", in Aparecida Vilaça et Robin Wright (éds), Natives christians. Modes and effects of christianity among indigenous peoples of the Americas, Ashgate Publishing, Farnham, pp. 127-146. 
CAPIBEribe Artionka

2007 Batismo de fogo. Os Palikur e o Cristianismo, Editora Annablume/Fapesp/ Nuti, São Paulo.

\section{Calavia Oscar}

2006 O nome e o tempo dos yaminawa. Etnologia e historia dos yaminawa do rio Acre, EdUnesp/Isa/Nuti, São Paulo.

Clastres Pierre

1974 La société contre l'État, Éditions de Minuit, Paris.

Descola Philippe

1987 La nature domestique. Symbolisme et praxis dans l'écologie des Achuar, Maison des sciences de l'homme, Paris.

1993 «Les affinités sélectives. Alliance, guerre et prédation dans l'ensemble jivaro », L'Homme, 33 (126-128), pp. 171-190.

ERIKSON Philippe

1986 «Altérité, tatouage et anthropophagie chez les Pano : la belliqueuse quête du soi », Journal de la société des Américanistes, LXII, pp. 185-210.

2009 "Obedient things : reflections on the Matis theory of materiality ", in Fernando Santos-Granero (éd.), The occult life of things. Native Amazonian theories of materiality, University of Arizona Press, Tucson.

\section{Fausto Carlos}

2001 Inimigos fieis. Historia, guerra e xamanismo na Amazonia, EdUSP, São Paulo.

FISHER William

2000 Rainforest exchanges. Industry and community on an amazonian frontier, Smithsonian Institution Press, Washington.

Freire Maria Jose A.

2001 A construção de um réu. Payakã e os Kayapo na imprensa durante a eco-92, dissertação de mestrado, Museu Nacional UFRJ, Rio de Janeiro.

Giannini Isabelle Vidal

1991 A ave resgatada. A impossibilidade da leveza do ser, dissertação de mestrado, Faculdade de Filosofia Letras e Ciências Humanas USP, São Paulo.

\section{Gordon Cesar}

2006 Economia selvagem. Ritual e mercadoria entre os indios Xikrin-Mebengokre, EdUnesp/Isa/Nuti, São Paulo.

2009 «Être féroce, être pacifique: des états affectifs comme expression de principes métaphysiques chez les Kayapo (Amazonie brésiliene) », exposé inédit fait au séminaire interne du Laboratoire d'Anthropologie Sociale, Collège de France, le 10 juin 2009, Paris, 22 p. [manuscrit].

HoFfMANN Hans

1964 "Money, ecology and acculturation among the Shipibo of Peru », in Ward H. Goodenough (éd.), Explorations in cultural anthropology. Essays in 
honour of George Peter Murdock, McGraw-Hill Book, New York/ San Francisco, pp. 259-276.

Howard Catherine

2002 "A domesticação de mercadorias : estrategias Waiwai », in Bruce Albert et Alcida Ramos (éds), Pacificando os brancos. Cosmologias do contato no norte-amazonico, Unesp-Imprensa Oficial do Estado, São Paulo, pp. 25-60.

Hugh-Jones Stephen

1988 "The gun and the bow : myths of white men and indians », L'Homme, 28 (106-107), pp. 138-155.

1992 "Yesterday's luxuries, tomorrow's necessities: business and barter in Northwest Amazonia ", in Caroline Humphrey et Stephen HughJones (éds), Barter, exchange and value, Cambridge University Press, Cambridge, pp. 42-74.

2009 «Patrimonialisation : entre image et écrit », conférence inédite donnée au séminaire ERSIPAL, Institut des hautes études de l'Amérique Latine, Paris.

KAPLAN Joanna Overing

1984 «Dualism as an expression of differences and danger: marriage exchange and reciprocity among the Piaroa of Venezuela », in Kenneth Kensinger (éd.), Marriage practices in Lowland South America, University of Illinois Press, coll. » Illinois studies in anthropology » 14, Urbana, pp. 127-155.

KeLly José Antonio

2005 "Notas para uma teoria do virar branco », Mana, 11 (1), pp. 201-234.

Lasmar Cristiane

2005 De volta ao lago de leite. Gênero e transformação no Alto Rio Negro, EdUnesp/Isa/Nuti, São Paulo.

LEA Vanessa

1986 Nomes e nekrets Kayapo. Uma concepção de riqueza, tese de doutorado, Museu Nacional UFRJ, Rio de Janeiro.

LÉvi-Strauss Claude

1973 "Le champ de l'anthropologie » (Leçon inaugurale au Collège de France), in Claude Lévi-Strauss, Antropologie structurale deux, Plon, Paris, pp. 11-44 [1960].

Lima Edilene Coffaci

2008 "As novas formas do kampo : elementos de uma sociologia da disseminação urbana dos saberes nativos ", in Marc Lenaerts et Ana Maria Spadafora (éds), Pueblos indigenas, plantas y mercados. Amazonia y Gran Chaco, Zeta Books, Bucharest, pp. 167-197.

Maybury-Lewis David (éd.)

1979 Dialetical societies. The Ge and Bororo of Central Brazil, Harvard University Press, Cambridge, Mass., Londres. 
Menget Patrick

1985 «Présentation », Journal de la Société des Américanistes, 71, pp. 129-130.

Mindein Betty

1985 Nos paiter: Os Surui de Rondônia, Editora Vozes, Petropolis.

OVERING Joanna

1992 "Wondering in the market and the forest : an Amazonian theory of production and exchange ", in Roy Dilley (éd.), Contesting markets, University of Edinburgh Press, Edinburgh, pp. 180-200.

RABBEN Linda

1998 Unnatural selection. The Yanomami, the Kayapó and the onslaught of civilization, University of Washington Press, Seattle.

SEeger Anthony

1981 Nature and society in Central Brazil. The Suyá Indians of Mato Grosso, Harvard University Press, Cambridge, Mass.

1993 «Ladrões, mitos e história : Karl von den Steinen entre os Suiás (3 a 6 de setembro de 1884) ", in Vera Penteado Coelho (éd.), Karl von den Steinen: um século de antropologia no Xingu, Edusp/FAPESP, São Paulo, pp. 431-444.

TURNER Terence

1992 «Os Mebengokre Kayapo: historia e mudança social, de comunidades autônomas para a coexistencia interétnica », in Manuela Carneiro da Cunha (éd.), Historia dos Indios no Brasil, Companhia das Letras/Fapesp, São Paulo, pp. 311-338.

1993 «De cosmologia a historia : resistência, adaptação e consciência social entre os Kayapo », in Eduardo Viveiros de Castro et Manuela Carneiro da Cunha (éds), Amazonia. Etnologia e história indigena, NHII/EdUSP/Fapesp, São Paulo, pp. 43-66.

1995 «An indigenous people's struggle for socially equitable and ecologically sustainable production : the Kayapo revolt against extractivism », Journal of Latin American Anthropology, 1 (1), pp. 98-121.

2009 «Valuables, value and commodities among the Kayapo of Central Brazil », in Fernando Santos-Granero (éd.), The occult life of things. Native amazonian theories of materiality, University of Arizona Press, Tucson.

Verswijver Gustaaf

1992 The club-fighters of the Amazon. Warfare among the Kayapo indians of Central Brazil, Rijksuniversiteit te Gent, Gent.

VILAÇA Aparecida

1992 Comendo como gente. Formas do canibalismo Wari, Editora da UFRJ, Rio de Janeiro.

2006 Quem somos nós. Os Wari' encontram os brancos, Editora UFRJ, Rio de Janeiro.

Viveiros de CAStro Eduardo

1992 From the enemy's point of view. Humanity and divinity in an Amazonian Society, Jorge Zahar/Anpocs, Rio de Janeiro. 
JOURNAL DE LA SOCIÉTÉ DES AMÉRICANISTES

\section{WERNER Denis}

1981 "Are some people more equal than others? Status inequality among the Mekranoti Indians of Central Brazil », Journal of Anthropological Research, 37 (4), pp. 360-373.

1982 "Leadership inheritance and acculturation among the Mekranoti of Central Brazil », Human Organization, 41 (4), pp. 342-344.

WILBERT Johannes (éd.)

1978 Folk literature of the Gê indians, UCLA Latin American Center Publications, Los Angeles. 